\title{
New NCCN Guidelines for Uveal Melanoma and Treatment of Recurrent or Progressive Distant Metastatic Melanoma
}

Presented by Christopher A. Barker, MD, and April K. Salama, MD

\section{Abstract}

The NCCN Guidelines Panel for Melanoma debuted new guidelines for uveal melanoma at the NCCN 23rd Annual Conference. Although uveal melanoma and cutaneous melanoma share the same name, they do have different characteristics and treatments. The NCCN Guidelines describe how tumor size guides therapeutic options, which for most tumors is radiotherapy. Predictors of melanoma-related mortality include advanced age, larger tumor size, and histopathologic and molecular features. The NCCN Guidelines for Cutaneous Melanoma have not changed notably, but adjuvant therapy with immunotherapies is now recommended. The best second-line treatment in the metastatic setting remains unclear.

J Natl Compr Canc Netw 2018;16(5.5):646-650 doi: 10.6004/jnccn.2018.0042

\section{New Guidelines Debut for Uveal Melanoma}

Uveal melanoma and cutaneous melanoma share a name, but they are very different cancers. The inaugural guidelines for the treatment of uveal melanoma were introduced at the NCCN 23rd Annual Conference by Christopher A. Barker, MD, Director of Clinical Investigation, Department of Radiation Oncology, Memorial Sloan Kettering Cancer Center.

"It's been recognized for some time that uveal melanoma is a distinct entity, and that we needed a set of guidelines pertaining to it," Dr. Barker said. "Several national guidelines exist for the management of uveal

Presented by Christopher A. Barker, MD, Department of Radiation Oncology, Memorial Sloan Kettering Cancer Center, New York, New York, and April K. Salama, MD, Division of Medical Oncology, Duke University Medical Center, Durham, North Carolina.

Dr. Barker has disclosed that he has received grant/research support from Amgen Inc., Elekta, Genentech, Inc., and Merck \& Co., Inc.; and has served as a scientific advisor for Pfizer Inc. Dr. Salama has disclosed that she has received grant/research support from Celldex, Dynavax

Technologies Corporation, Genentech, Inc., Bristol-Myers Squibb

Company, Immunocore, Merck \& Co., Inc., and Reata; has served as a scientific advisor for Array and Merck \& Co., Inc.; has received consulting fees/honoraria from Biocryst and Bristol-Myers Squibb

Company; and has served on the product/speakers' bureau for Bristol-

Myers Squibb Company. Her spouse has received grant/research support from AbbVie.

Correspondence: Christopher A. Barker, MD, Department of Radiation Oncology, Memorial Sloan Kettering Cancer Center, 1275 York Avenue,

Box 22, New York, NY 10065. Email: barkerc@mskcc.org; and

April K. Salama, MD, Division of Medical Oncology, Duke University

School of Medicine, DUMC 3198, Durham, NC 27710.

Email: april.salama@duke.edu melanoma; however, none provide a pathway-based strategy for the management of patients with all stages of disease. These new NCCN Guidelines for the management of uveal melanoma are the first pathway-based guidelines to be developed."

Also at the conference, April K. Salama, MD, Assistant Professor, Division of Medical Oncology, Duke University Medical Center, described the state of the art in treating the more common cutaneous melanoma, focusing much of her talk on the uncertainties of second-line options. "This year, we are just highlighting that there are limited data to make second-line therapy recommendations. We are largely extrapolating based on first-line data," she noted.

\section{Uveal Melanoma: How It's Different}

Primary treatment for uveal melanoma is based on tumor size and is commonly radiotherapy (RT) rather than surgery. As with cutaneous melanoma, mutations are important but molecular testing is prognostic rather than predictive. Recurrences are mostly at distant metastatic sites—specifically the liver-rather than the skin or lymph nodes.

Uveal melanoma occurs in approximately 1 per 200,000 Americans annually. ${ }^{1}$ Median age at diagnosis is 60 years, with the predominant disease site being 
NCCN Guidelines for Melanoma

the choroid. Risk factors for this malignancy include the presence of choroidal nevi, presence of ocular or oculodermal melanocytosis, and familial syndromes, including germline BAP1 mutations, neurofibromatosis, and dysplastic nevus syndrome. Importantly, cutaneous melanoma is not a risk factor for uveal melanoma, Dr. Barker indicated.

\section{Clinical Presentation and Workup}

Approximately two-thirds of patients present due to changes in their vision; approximately a third are asymptomatic and the disease is discovered on routine eye examination. A comprehensive eye examination is absolutely vital to the evaluation and staging of patients, and this is detailed in the NCCN Guidelines recommendations.

"Biopsy is typically not performed to confirm diagnosis unless the clinical diagnosis is in question. However, a biopsy may be performed for prognostic purposes through molecular testing," Dr. Barker said.

There are 2 primary staging systems for uveal melanoma, and both are based on tumor height and largest base diameter. The first staging system was the Collaborative Ocular Melanoma Study (COMS), followed by the AJCC staging system. In the COMS staging system, tumors are described as either small, medium, or large, whereas in the AJCC system they are staged as T1, T2, T3, or T4. Some overlap exists between the COMS small and medium categories and the AJCC T1 and T2 categories, and between the COMS large category and AJCC T3 and T4 categories. The AJCC system also incorporates the extent of primary tumor involvement through T-stage modifiers. Presence of ciliary body involvement or extraocular extension of the primary tumor is associated with T-stage modifiers A, B, C, D, or E.

\section{COMS Trials Inform Treatment}

The COMS was a series of landmark trials that helped define the management of primary uveal melanoma (Figure 1). These findings have informed the new NCCN Guidelines for Uveal Melanoma.

The first COMS study, conducted in the late 1980s, elucidated the natural history of small uveal melanomas and determined the safety of observation, with treatment at the time of tumor growth. The second trial of medium-sized tumors randomized patients to definitive plaque brachytherapy or enucleation, the standard treatment. The third trial randomized patients with large uveal melanomas to

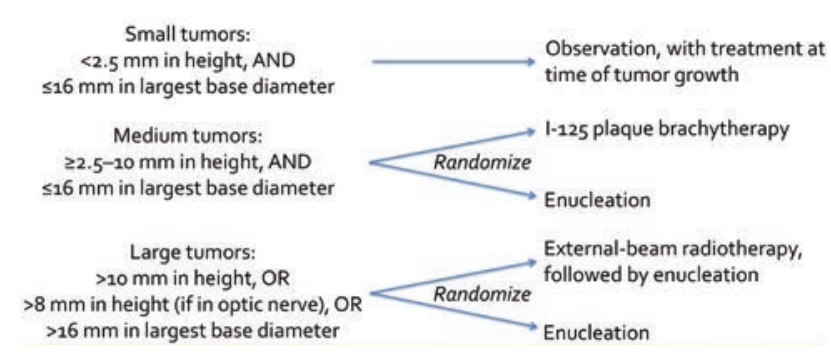

Figure 1. The Collaborative Ocular Melanoma Study (COMS) helped define primary tumor management.

neoadjuvant preoperative external RT to the orbit followed by enucleation, or enucleation alone.

The COMS small tumor trial showed that melanoma-related mortality was low for this subset $(<4 \%$ approximately 8 years after diagnosis) and that the growth of small uveal melanomas is rare $(\approx 30 \%$ at 5 years). ${ }^{2}$ Characteristics that predicted for growth are programmed into the risk assessment of these patients. ${ }^{3}$ Tumors that grow are primarily treated with RT or laser therapy.

The COMS medium tumor trial, conducted during a 10-year span, found no difference in overall survival between patients randomized to definitive plaque brachytherapy or enucleation, and no difference in cancer mortality. ${ }^{4}$ Survival at 5 years was approximately $80 \%$, and approached $60 \%$ at 12 years. Predictors of melanoma-related mortality included advanced age and larger tumor size.

"A series of investigations found that quality of life, however, was different between the 2 groups," ${ }^{5}$ Dr. Barker added. "Patients preserved vision if they underwent brachytherapy versus enucleation. However, patients treated with brachytherapy tended to have more anxiety than those who underwent enucleation."

Finally, the COMS large tumor trial found no difference in survival between neoadjuvant RT followed by enucleation, or enucleation alone ${ }^{6} ; 5$-year survival was approximately $60 \%$ and 10 -year survival approached $40 \%$. Again, predictors of melanomaspecific mortality included advanced age and larger tumors. The study did find a lower risk for orbital recurrence in patients who received neoadjuvant RT, but this difference was not clinically significant; thus, neoadjuvant RT is not part of standard management. Particle beam therapy may be an alternative to enucleation in select patients with large tumors, but this was not studied by the COMS group. 
Management of primary uveal melanoma is predominantly based on tumor size. The NCCN Guidelines give several options for any given size tumor, generally including RT, laser therapy, and surgical removal, with some additional adjuvant options in select situations.

"In this day and age, enucleation is only being performed for large tumors," Dr. Barker indicated. As for risk assessment, he said the data are insufficient for the use of composite measures, therefore, "we generally look at variables in isolation."

\section{Genomics and Other Factors Associated With Risk of Metastasis}

Cutaneous and uveal melanomas are different genetically in that cutaneous disease harbors far more mutations, and these are different from those in uveal melanoma. "As I mentioned, genomic testing is predictive in cutaneous melanoma. For instance, the BRAF mutation is a predictor of response to RAF inhibition. In addition, the overall number of mutations seen in cutaneous melanoma may be a predictor of the response to therapy," Dr. Barker noted. "On the other hand, in uveal melanomas, specific genomic alterations are not associated with response to treatment, but they are associated with prognosis and the probability of metastasis."

Along with gene expression profile and specific genetic alterations (eg, BAP1 is associated with worse survival than EIFAX1, which is associated with virtually no risk), other risk factors for metastasis include certain clinical factors ( $\mathrm{T}$ stage and T-stage modifiers, ciliary body involvement, extraocular extension), histopathologic factors (spindle vs epithelioid cell type), and cytogenetics (status of chromosomes 3, 5, and 8). These factors can be used in risk stratification.

\section{Follow-Up Imaging and Treatment of Metastases}

Although whole-body imaging is recommended for surveillance in patients with high-risk cutaneous melanoma, uveal melanoma recurrence most often occurs in the liver (Figure 2). For this reason, surveillance of patients with high-risk uveal melanoma should include specific imaging of the liver.

"The NCCN Guidelines recommend surveillance according to risk for metastasis. These risk groups help clinicians identify how often imaging should be performed in their surveillance strategy," Dr. Barker stated.
For distant metastasis, no single systemic therapy has proven to be most effective for uveal melanoma. Thus, the NCCN Guidelines encourage clinical trial participation when possible. Although drugs that are effective in cutaneous melanoma are far less effective for uveal disease, they can still be considered. Liverdirected therapy is also part of management, which includes resection, ablation, chemoembolization, radioembolization, and regional perfusion.

\section{Current Treatment of Cutaneous Melanoma}

Dr. Salama reviewed the current treatment approaches to cutaneous melanoma, noting that although the field has rapidly improved outcomes in the first-line setting for advanced melanoma, there are less data for second-line treatment. She also noted that immunotherapies that are robust in the advanced disease setting are now proving their worth as adjuvant treatment.

Several effective options are now approved for adjuvant therapy besides observation. Nivolum$\mathrm{ab}$ is now preferred over ipilimumab for resected stage IIIB/C melanoma. For patients with sentinel node metastasis $>1 \mathrm{~mm}$, the NCCN Guidelines recommend dabrafenib/trametinib (for patients with BRAF V600-mutated disease), in addition to nivolumab.

\section{Second-Line Therapies for Advanced Disease}

With the approval of PD-1 checkpoint inhibitors and targeted agents for first-line treatment of advanced/metastatic disease, the direction for secondline therapy is less certain because most of the data for these agents come from studies of previously untreated patients.

"Despite the rapid pace of development of novel therapies for melanoma, for second-line treatment of advanced disease it doesn't take long to feel we are back at the beginning-with not many options and questionable choices," she said.

Dr. Salama then listed several factors to consider when selecting second-line therapy, including disease characteristics (eg, mutation status of BRAF, and perhaps NRAS and KIT), prior therapies (eg, response, setting, and timing of relapse), and prior toxicities. "Generally, you can switch classes. Sometimes you can go back to the same agent, particularly if there has been a long disease-free interval or lack 

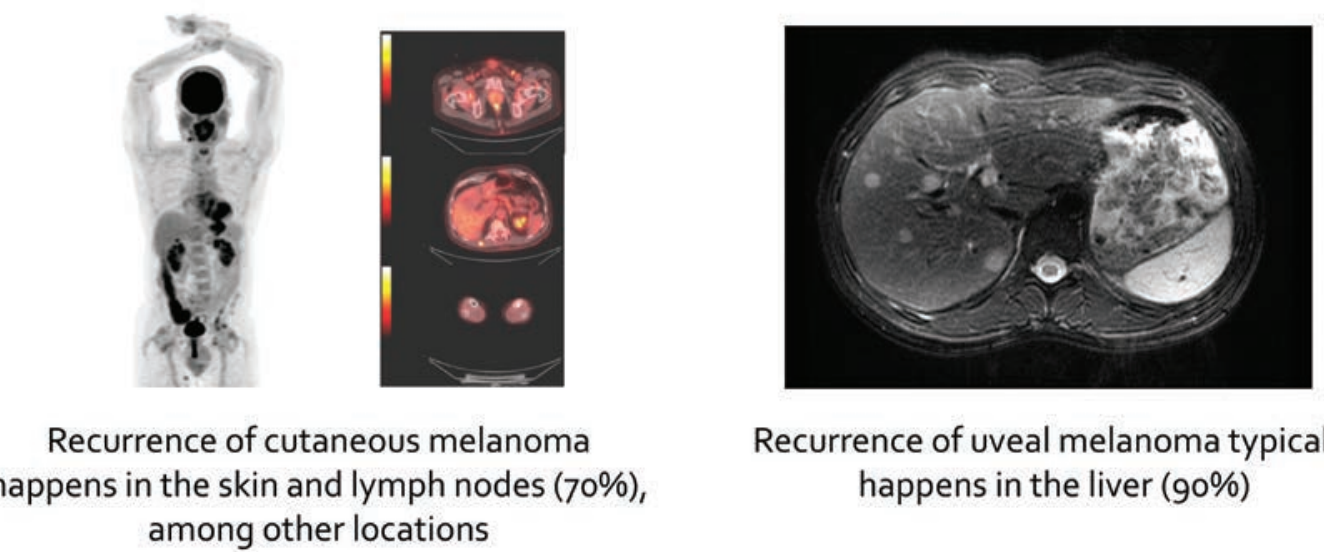
Recurrence of uveal melanoma typically happens in the liver (90\%)

Figure 2. Follow-up imaging after treatment of the primary tumor is based on most likely site of metastatic recurrence.

of progression for some time. With prior toxicities, especially if they were severe, retreating with the same agent or class may be challenging."

Among the systemic therapy options in the second or subsequent line of treatment are anti-PD-1 as monotherapy if not given for initial treatment (ie, nivolumab, pembrolizumab); ipilimumab alone or with nivolumab; targeted therapy based on BRAF or KIT mutation status; high-dose interleukin-2; and best supportive care.

\section{Checkpoint Inhibitors in the Second Line}

"Many patients have already received anti-PD-1 in the frontline," Dr. Salama noted. "We've tried to list many of the same agents as for the frontline, highlighting the issue of switching class if possible and potentially looking at other agents."

It is reasonable for patients with a good performance status progressing on anti-PD-1 therapy to consider dual checkpoint inhibitors. If at least 3 months have elapsed since frontline therapy was discontinued, reintroduction of the same agent or class should be considered, the NCCN Guidelines state.

Prospective data in the frontline setting are very limited, but pembrolizumab after progression on ipilimumab has shown some benefit.? "However, now that patients receive anti-PD-1 agents in the frontline, we have no [relevant] data for the second-line setting," she continued. "What is available are some retrospective series, overall with small numbers of patients."

Some retrospective studies have found modest benefit for ipilimumab after anti-PD-1, and perhaps slightly higher activity for the combination of ipili- mumab and anti-PD-1. Although the response rate is approximately $60 \%$ in the first-line setting, these series suggest that the activity of dual checkpoint inhibition is diminished in the second-line setting, Dr. Salama said.

Another concern is increased toxicity with dual therapy, which appears to be similar irrespective of disease setting. In a study from Memorial Sloan Kettering Cancer Center, 91\% of patients on ipilimumab/nivolumab had a clinically significant adverse event, two-thirds sought emergency medical care, and many were hospitalized. ${ }^{8}$

\section{Targeted Therapies in the Second Line}

Sequencing also involves targeted agents, and again, the data for guidance are scarce. In a study of 274 patients with BRAF-mutated metastatic melanoma, those receiving a BRAF inhibitor in the frontline and ipilimumab in the second-line had no clinical responses. ${ }^{9}$ More recent studies have evaluated sequencing with anti-PD-1 agents. In a retrospective study of 114 patients, no differences in survival were shown between patients receiving either BRAF/ MEK-targeted therapy or anti-PD-1 agents first, but subsequent anti-PD-1 therapy given after ipilimumab showed some evidence of diminished efficacy versus the reverse, Dr. Salama said. ${ }^{10}$

Regarding choice of drugs and whether they should be given in combination or sequenced, there are still no definitive directives. Currently, agents being investigated in clinical trials include concurrent BRAF/MEK inhibition plus an anti-PD-1 or the sequential administration of an BRAF/MEK inhibitor before an anti-PD-1 (+ ipilimumab) or the reverse. 
"We probably have more questions than answers at this point," Dr. Salama acknowledged.

To help determine the best sequence for BRAFmutated metastatic melanoma, the randomized phase III ECOG-ACRIN EA6134 study will compare dabrafenib/trametinib and ipilimumab/nivolumab on progression, and the same in reverse sequence (ClinicalTrials.gov identifier: NCT02224781).

\section{Extrapolating From the First-Line Setting}

"Meanwhile, the choice of second-line therapy is largely extrapolated from the first-line setting," Dr. Salama concluded (Figure 3). "There's no perfect treatment...switching the class of agent is usually the best course. The main factors we now consider are clinical when selecting a therapy. No perfect therapy exists, even in the frontline. Based on all these factors, we have to choose a therapy that best fits patients' clinical needs."

Anti-PD-1 monotherapy produces high response rates but can take some time to work; responses can

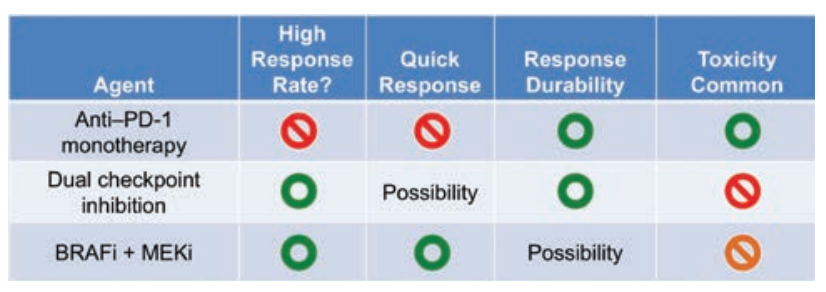

Figure 3. How to choose therapy in 2018? Largely extrapolated from the first-line setting.

be durable, and the toxicity profile is favorable. Dual checkpoint inhibition produces higher response rates, although these are diminished in the secondline setting; fast responses are possible and are often durable, but toxicity can be an issue. In Dr. Salama's experience, BRAF/MEK inhibition alone is the most reliable in terms of initial response; although durable responses are possible, many patients progress. Serious, life-threatening toxicities are uncommon, though chronic toxicities can be problematic, she suggested.

\section{References}

1. Singh AD, Turell ME, Topham AK. Uveal melanoma: trends in incidence, treatment, and survival. Ophthalmology 2011;118:1881-1885.

2. Mortality in patients with small choroidal melanoma. COMS report No. 4. Collaborative Ocular Melanoma Study Group. Arch Ophthalmol 1997;115:886-893

3. Factors predictive of growth and treatment of small choroidal melanoma: COMS report No. 5. The Collaborative Ocular Melanoma Study Group. Arch Ophthalmol 1997;115:1537-1544.

4. Collaborative Ocular Melanoma Study Group. The COMS randomized trial of iodine 125 brachytherapy for choroidal melanoma: V. Twelveyear mortality rates and prognostic factors: COMS report No. 28. Arch Ophthalmol 2006;124:1684-1693.

5. Melia M, Moy CS, Reynolds SM, et al. Quality of life after iodine 125 brachytherapy vs enucleation for choroidal melanoma: 5 -year results from the Collaborative Ocular Melanoma Study: COMS QOLS report No. 3. Arch Ophthalmol 2006;124:226-238.

6. Hawkins BS; Collaborative Ocular Melanoma Study Group. The Collaborative Ocular Melanoma Study (COMS) randomized trial of pre-enucleation radiation of large choroidal melanoma: IV. Ten-yea mortality findings and prognostic factors. COMS report number 24. Am J Ophthalmol 2004;138:936-951.

7. Ribas A, Puzanov I, Dummer R, et al. Pembrolizumab versus investigator-choice chemotherapy for ipilimumab-refractory melanoma (KEYNOTE-002): a randomized, controlled, phase 2 trial. Lancet Oncol 2015;16:908-918

8. Friedman CF, Navid-Azarbaijani P, Shoushtari AN, et al. Toxicity associated with ipilimumab and nivolumab (Ipi+Nivo) combination therapy in melanoma patients (pts) treated at a single institution under an expanded-access program (EAP) [abstract]. J Clin Oncol 2016;34(Suppl15):Abstract 9519.

9. Johnson DB, Pectasides E, Feld E, et al. Sequencing treatment in BRAFV600 mutant melanoma: anti-pd-1 before and after BRAF inhibition. J Immunotherapy 2017;40:31-35.

10. Ackerman A, Klein O, McDermott DF, et al. Outcomes of patients with metastatic melanoma treated with immunotherapy prior to or after BRAF inhibitors. Cancer 2014;120:1695-1701. 